\title{
MATCHING DIVER AND EQUIPMENT
}

\author{
Professor A. S. G. Curtis
}

Departiment of Cell Biology, University of Glasgow

Efficient diving requires the following:

i) Personnel whose physiological and psychological make up is sufficient to meet the stresses of diving.

ii) Equipment that is good enough to be able to meet the physiological requirements of breathing, heat gain or loss etc., of the diver.

iii) Adequate training systems.

All three criteria require selection procedures if they are to be used effectively. The physiological matching of the diver to his equipment and vice-versa, shall be considered leaving the questions of psychological stress and selection for trainability and actual training to others.

We are fairly well informed on the desirable partial pressures of oxygen and carbon dioxide that must be present in the inhalant and exhalant gas, Miles (1969), Penzias and Goodman (1973). There is a similarly good knowledge of the role of inert gases. However there is very little work to provide answers to the following problems.

1. How will flow rates in the human airways vary with airway diameter and length, turbulence, pressure drop, and gas density and viscosity? Under what conditions will ventilation, particularly maximal ventilation become insufficient to prevent hypoxia or hypercapnia?

2. How great a power output can be used by the average healthy human in prolonged respiration requiring great exertion? Can gas density and viscosity be so great and turbulence driving be such a sink of power that the diver is unable to maintain respiration?

3. What effects will result from adding on breathing apparatus which will increase the power requirements and tend to decrease flow rates? How can this equipment be designed to minimise these unfortunate effects?

A simple physical analysis of gas transport in pipes, though very theoretical, tells us that gas flow in the airways of a human is probably largely laminar at surface pressure but that increasing gas density in particular, which occurs of course with increasing pressure, is sufficient to bring the system to the point of turbulence or to markedly enlarge any regions of turbulence that already existed at the surface.

The increase in turbulence means that the power input to sustain a certain ventilatory volume per minute rises sharply. It should of course be added that at the surface maximum flow rates are "effort independent" but this does not apply to volume per minute at increasing depth. The increased gas density, in addition to its effects on the onset and spread of turbulence, also directly increases the power requirement to sustain a certain gas ventilation rate. Uhl (1971) suggests that the healthy diver cannot sustain a respiratory power input exceeding $11 \mathrm{~kg} \mathrm{M}^{-1} \mathrm{~min}$. Unfortunately there appear to be insufficient measurements on the respiratory power requirements of divers at depth to give us any great knowledge of the precise conditions [i.e. gas mixtures composition, pressure] at which these limits are reached for the average healthy diver. We are even less certain as to what tolerances in respiratory power may exist in human beings and how stable these are. Morrison (1973) in two interesting studies has shown (i) that divers using SCUBA equipment have a maximal ventilatory volume which diminishes rapidly with increasing depth, and (ii) that RN divers using CDBA apparatus are close to the limit of hypoxia and hypercapnia in using this equipment when maximum ventilation is required, Morrison and Butt (1972). Unfortunately in these studies the effects of pressure have not been separated from those arising from the actual breathing equipment used.

Vail (1971) has suggested that airway collapse will tend to occur at depth simply because the intrathoracic airway pressure drop may tend to become very great at depth. He suggests that the subsegmental bronchi will be the prime site of this collapse. We do not know in practice whether this actually occurs at depth, but if it does this would reduce gas exchange (and the collapse might provide a possible explanation of some cases of air embolism). The addition of breathing equipment to the respiratory pathway might set up conditions precipitating this collapse under conditions that would otherwise be satisfactory. Vail goes on to suggest that the resulting carbon dioxide accumulation is entirely responsible for the symptoms of inert gas narcosis: but I feel that there is much evidence against this final feature of Vail's thesis.

Equipment increases the work load, simply because 
the resistance to gas flow increases due to the relatively narrow diameter of the gas supply and exhalant tubes, use of valves and of absorbent granule beds (in certain types of equipment). It is clear that in designing equipment the following criteria should be met if possible.

(i) Resistance to exhalation less than $3 \mathrm{~cm} \mathrm{H}_{2} \mathrm{O}$ at all depths.

(ii) Minimum gas density at any given depth, compatible with biochemical requirements. This requirement is one of the reasons for the advantages of helium in gas mixtures.

(iii) Airways with minimum deadspace compatible with sufficient width for low resistance, and an absence of turbulence-producing features.

(iv) The equipment should meet these requirements whatever the physical attitude of the diver.

(v) Deadspace should be minimal to prevent hypoxia and hypercapnia.

Uhl (1972) tried increasing the respiratory work loading of divers by changing gas density and found that work and power requirements are likely to be the chief limiting factor on respiration at depth.

It is clear that we have little physiological knowledge on which designers of equipment may draw when we are considering the problems of gas exchange. To a considerable extent at present, equipment is designed partly on simple rule of thumb concepts and partly on the related principle that if the diver likes it it must be all right. These principles may work well in many situations yet fail unexpectedly in slightly different but novel ones. More research is needed in this area to provide safer diving equipment.

\section{REFERENCES}

MiiLES, S. (1969). Underwater Medicine. Staples. London.

MORRISON, J. B. and BUTT, W. S. (1972). Effect of underwater breathing apparatus and absolute air pressure on divers' ventilatory capacity. Aerosp. Med. 43: 881-6.

MORRISON, J. B. (1973). Oxygen uptake studies of divers when fin swimming with maximum effort at depths of 6-176 feet. Aerosp.Med. 44: 1120-9.

PENZIAS, W. and GOODMAN, M. W. (1973). Man beneath the sea. Wiley. London, New York.

UHL, R. R. et al (1972). Effects of externally imposed mechanical resistance on breathing dense gas at exercise: mechanics of breathing. Aerosp.Med. 43: 836-41.

VAIL, E. G. (1971). Hyperbaric respiratory mechanics. Aerosp.Med. 42: 536-46. 